\title{
Increase in Hospital Admissions for Severe Influenza A/B among Travelers on Cruise Ships to Alaska, 2015
}

\section{Michael Payne, Danuta Skowronski, Suzana Sabaiduc, Linda Merrick, Christopher Lowe}

An increase in hospital admissions for influenza occurred during the summer of 2015 at an acute care facility in Vancouver, British Columbia, Canada. Investigation identified 25 patients with recent history of cruise ship travel to Alaska. All characterized influenza A viruses were A(H3N2). We describe patient treatment regimens and outcomes.

$\mathrm{D}$ sorders of the respiratory tract are the most common cause of medically attended illness (29\%) among cruise ship passengers and crew (1). Influenza outbreaks associated with travel on cruise ships, including those sailing to Alaska in the summer months (2-5), have been described previously. The number of travelers on cruises to Alaska is large, with $\approx 900,000$ documented in 2011 (6). We report an increase in hospital admissions for severe influenza among travelers on cruise ships to Alaska during the summer of 2015 at an acute care facility in Vancouver, British Columbia, Canada.

\section{The Study}

During the 2015 summer season (June-September), an increase in admissions for severe influenza was detected at our hospital, despite minimal influenza activity in the community $(7,8)$. We determined that 25 cruise ship travelers to Alaska tested positive by PCR for influenza at hospital admission, 24 for influenza $\mathrm{A}$ and 1 for influenza $\mathrm{B}$. We included all 25 of these patients in our study. We reviewed patient charts to determine date of illness onset, vaccination status, risk factors, treatment with antivirals or antibiotics, and patient outcomes (9). We sent influenza A-positive samples to the provincial public health reference laboratory for typing and performed gene sequencing on an additional subset to establish phylogenetic clade.

Author affiliations: Providence Health Care, Vancouver, British Columbia, Canada (M. Payne, L. Merrick, C. Lowe); University of British Columbia, Vancouver (M. Payne, D. Skowronski, C. Lowe); BC Centre for Disease Control Public Health Laboratory,

Vancouver (D. Skowronski, S. Sabaiduc)

DOI: https://doi.org/10.3201/eid2403.171378
The study group consisted of 11 women and 14 men (average age 72.5 years). Twenty-two travelers were from the United States, 2 from Canada, and 1 from the United Kingdom. Only 4 patients had receipt of the 2014-15 influenza vaccine documented in their medical chart. All patients had $\geq 1$ risk factor for severe influenza, with an average of 2.5 recognized risk factors per patient. The most common were age $\geq 65$ years ( $84 \%$ ), cardiovascular disease (48\%), and pulmonary disease (44\%). Symptom onset occurred on average 4.1 days before admission. Eight patients were documented to have an earlier influenza diagnostic test performed on board the ship; 7 were positive, and 1 was negative. All 7 of these influenza-positive patients were started on oseltamivir before hospital admission. However, none of the patients who were negative or not tested for influenza on the cruise ship were documented to have been empirically treated with oseltamivir. Before admission, antibiotics were prescribed to 12 patients, with an average course lasting 2.2 days.

Admission blood culture results were negative for all 25 patients. Admission sputum cultures were collected from 17 patients: 1 showed heavy growth of methicillinresistant Staphylococcus aureus, and the other 16 showed no growth or normal respiratory flora. During hospitalization, oseltamivir treatment was administered to 19 patients, with average duration (including discharge prescriptions) of 6.1 days. Twenty-two patients were empirically started on antibiotics at hospital admission. The average length of antibiotic treatment, including discharge prescriptions, was 7.4 days from the date of admission.

In-hospital complications included 1 case of Pseudomonas aeruginosa ventilator-associated pneumonia, 1 myocardial infarction, and 1 tracheostomy. No inhospital deaths occurred. The average length of hospitalization was 7.1 days. Patients with influenza diagnosed on the cruise ship and started on oseltamivir $(n=7)$ had an average length of stay of 5.3 days versus 7.8 days for patients with influenza diagnosed in hospital only $(\mathrm{n}=$ 18), but this difference was not statistically significant ( $\mathrm{p}$ $=0.42$ by 2 -tailed $t$-test).

All influenza A virus samples were sent to a reference laboratory for subtyping and were identified as H3N2. In addition, 16/24 isolates underwent gene sequencing and were identified as clade 3 C.2a, the dominant genetic 
and antigenic drift variant responsible for the 2014-15 influenza epidemic in the Northern Hemisphere. Clade $3 \mathrm{C} .2 \mathrm{a}$ viruses are distinguished from the 2014-15 A/ Texas/50/2012 (clade 3C.1) vaccine strain by multiple (10-12) amino acid substitutions at antigenic sites of the surface hemagglutinin protein, notably F159Y and adjacent K160T mutations within immunodominant antigenic site $\mathrm{B}$, the latter conferring a potential gain of glycosylation further relevant to antibody binding $(10,11)$. The antigenic drift of clade $3 \mathrm{C} .2 \mathrm{a}$ viruses and their mismatch to the 2014-15 vaccine were widely recognized that season, with historically low vaccine effectiveness reported and recommendations issued for adjunct protective measures (11).

\section{Conclusions}

We present a review of 25 cases of laboratory-confirmed influenza illness requiring hospital admission during the summer of 2015 and associated with cruise ship travel to Alaska. The study was initiated in response to the perception by hospital staff of an atypical surge in admissions for severe influenza in summer 2015, with 27 of 33 influenza A-positive patients having illness associated with cruise ship travel, compared with 1 of 3 cases in summer 2014 and 8 of 19 cases in summer 2016.

The US Centers for Disease Control and Prevention (CDC) has published guidelines for the management of influenza-like illness (ILI) on cruise ships (12). For prevention, $\mathrm{CDC}$ recommends that crew and passengers receive the season's recommended influenza vaccination, postpone travel when ill, and comply with respiratory etiquette and that persons who are ill be appropriately isolated. In addition, CDC recommends antiviral treatment in cases of confirmed or suspected influenza in ILI patients with severe manifestations or risk factors for severe disease. Cruise ship passengers, particularly those at high risk for severe influenza complications, should be advised of these recommended measures before travel to mitigate their risk.

In our review, 7 patients had influenza A diagnosed on the cruise ship; 1 patient had tested negative for influenza A/B on the ship. Only patients who tested positive for influenza $\mathrm{A}$ on the cruise ship were documented to have been started on oseltamivir; however, a positive test is not required for initiation of treatment, as per CDC guidelines, especially in the context of a documented outbreak (12). Point-of-care influenza A/B antigen tests have poor sensitivity; therefore, in the appropriate clinical scenario, treatment should not be withheld on the basis of a negative test (13). In this small case series, patients who received treatment with oseltamivir before admission had a shorter duration of stay $(\Delta$ $=2$ days), although this difference was not statistically significant. Definitive conclusions cannot be drawn from this observation, although it corresponds with a previous metaanalysis that noted a $21 \%$ decrease in the time to alleviation of symptoms in the oseltamivir treatment versus placebo group (14).

Our case series has several limitations. First, minimal follow-up information was available after discharge from hospital. Second, all of our patients had countries of origin in the Northern Hemisphere, but propagated viruses might have been introduced from elsewhere. Cruise ships to Alaska include travelers from the Southern Hemisphere, where influenza peaks during JuneSeptember. Third, we relied on the cruise ship medical records to provide information on treatment before admission. For many patients, this information was not provided, and if so, we assumed that no treatment or further diagnostics occurred. Finally, our review focused on a single facility; patients admitted to other facilities in the region would add to the total disease burden but were not captured in this study.

In summary, we report a series of severe influenza cases requiring hospitalization among cruise ship travelers to Alaska during the summer of 2015. Cruise ship passengers should be advised of such influenza risks and preventive measures before travel. Our findings reinforce the need for surveillance monitoring to inform timely initiation of antiviral treatment during cruise ship outbreaks. Clinicians caring for passengers with ILI should consider empiric influenza therapy, particularly because cruise ship travelers can include a large proportion of persons at risk of severe influenza complications.

\section{Acknowledgments}

We thank the laboratory technologists at Providence Health Care and the BC Centre for Disease Control for their work on this study. Also, we thank the National Microbiology Laboratory of Canada for their contributions to the influenza virus typing. Finally, we thank Reka Gustafson and Mel Krajden.

\section{About the Author}

Dr. Payne is a medical microbiologist with Providence Health Care in Vancouver, British Columbia. His research interests include molecular diagnostics and travel and tropical medicine.

\section{References}

1. Peake DE, Gray CL, Ludwig MR, Hill CD. Descriptive epidemiology of injury and illness among cruise ship passengers. Ann Emerg Med. 1999;33:67-72. http://dx.doi.org/10.1016/ S0196-0644(99)70419-1

2. Uyeki TM, Zane SB, Bodnar UR, Fielding KL, Buxton JA, Miller JM, et al.; Alaska/Yukon Territory Respiratory Outbreak 
Investigation Team. Large summertime influenza A outbreak among tourists in Alaska and the Yukon Territory. Clin Infect Dis. 2003;36:1095-102. http://dx.doi.org/10.1086/374053

3. Brotherton JM, Delpech VC, Gilbert GL, Hatzi S, Paraskevopoulos PD, McAnulty JM; Cruise Ship Outbreak Investigation Team. A large outbreak of influenza A and B on a cruise ship causing widespread morbidity. Epidemiol Infect. 2003;130:263-71. http://dx.doi.org/10.1017/ S0950268802008166

4. Millman AJ, Kornylo Duong K, Lafond K, Green NM, Lippold SA, Jhung MA. Influenza outbreaks among passengers and crew on two cruise ships: a recent account of preparedness and response to an ever-present challenge. J Travel Med. 2015;22:30611. http://dx.doi.org/10.1111/jtm.12215

5. Ward KA, Armstrong P, McAnulty JM, Iwasenko JM, Dwyer DE. Outbreaks of pandemic (H1N1) 2009 and seasonal influenza A (H3N2) on cruise ship. Emerg Infect Dis. 2010;16:1731-7. http://dx.doi.org/10.3201/eid1611.100477

6. US Department of Transportation Maritime Administration. North American cruise statistical snapshot, 2011 [cited 2017 Aug 15]. https://www.marad.dot.gov/wp-content/uploads/pdf/North American_Cruise_Statistics_Quarterly_Snapshot.pdf

7. Guiyun Li $\bar{H}$, Chambers C, Kwindt L, Skowronski D. British Columbia influenza surveillance bulletin: influenza season 2014-15, number 22, week 38, September 18 to 24, 2016 [cited 2017 Aug 15]. http://www.bccdc.ca/resource-gallery/Documents/ Statistics\%20and\%20Research/Statistics\%20and\%20Reports/ Epid/Influenza\%20and\%20Respiratory/2015-2016/ InfluBulletin_Number22_Week38_201516.pdf

8. State of Alaska Epidemiology. Alaska influenza surveillance summary, 2014-15 season [cited 2017 Aug 15]. http://epibulletins.dhss.alaska.gov/Document/ Display?DocumentId $=32$

9. Aoki FY, Allen UD, Stiver HG, Evans GA. The use of antiviral drugs for influenza: guidance for practitioners 2012/ 2013. Can J Infect Dis Med Microbiol. 2012;23:e79-92. http://dx.doi.org/10.1155/2012/879141

10. Skowronski DM, Sabaiduc S, Chambers C, Eshaghi A, Gubbay JB, Krajden M, et al. Mutations acquired during cell culture isolation may affect antigenic characterisation of influenza A(H3N2) clade 3C.2a viruses. Euro Surveill. 2016;21:30112. http://dx.doi.org/10.2807/1560-7917. ES.2016.21.3.30112

11. Skowronski DM, Chambers C, Sabaiduc S, De Serres G, Winter AL, Dickinson JA, et al. A perfect storm: impact of genomic variation and serial vaccination on low influenza vaccine effectiveness during the 2014-15 season. Clin Infect Dis. 2016;63:21-32. http://dx.doi.org/10.1093/cid/ciw176

12. US Centers for Disease Control and Prevention. Guidance for cruise ships on influenza-like illness (ILI) management [cited 2017 Aug 15]. http://www.cdc.gov/quarantine/cruise/management/ guidance-cruise-ships-influenza-updated.html

13. Chartrand C, Leeflang MM, Minion J, Brewer T, Pai M. Accuracy of rapid influenza diagnostic tests: a meta-analysis. Ann Intern Med. 2012;156:500-11. http://dx.doi.org/10.7326/ 0003-4819-156-7-201204030-00403

14. Dobson J, Whitley RJ, Pocock S, Monto AS. Oseltamivir treatment for influenza in adults: a meta-analysis of randomised controlled trials. Lancet. 2015;385:1729-37. http://dx.doi.org/10.1016/ S0140-6736(14)62449-1

Address for correspondence: Michael Payne, St. Paul's Hospital,

Department of Microbiology, 1081 Burrard St, Vancouver, BC V6Z 1Y6, Canada; email: mpayne@providencehealth.bc.ca

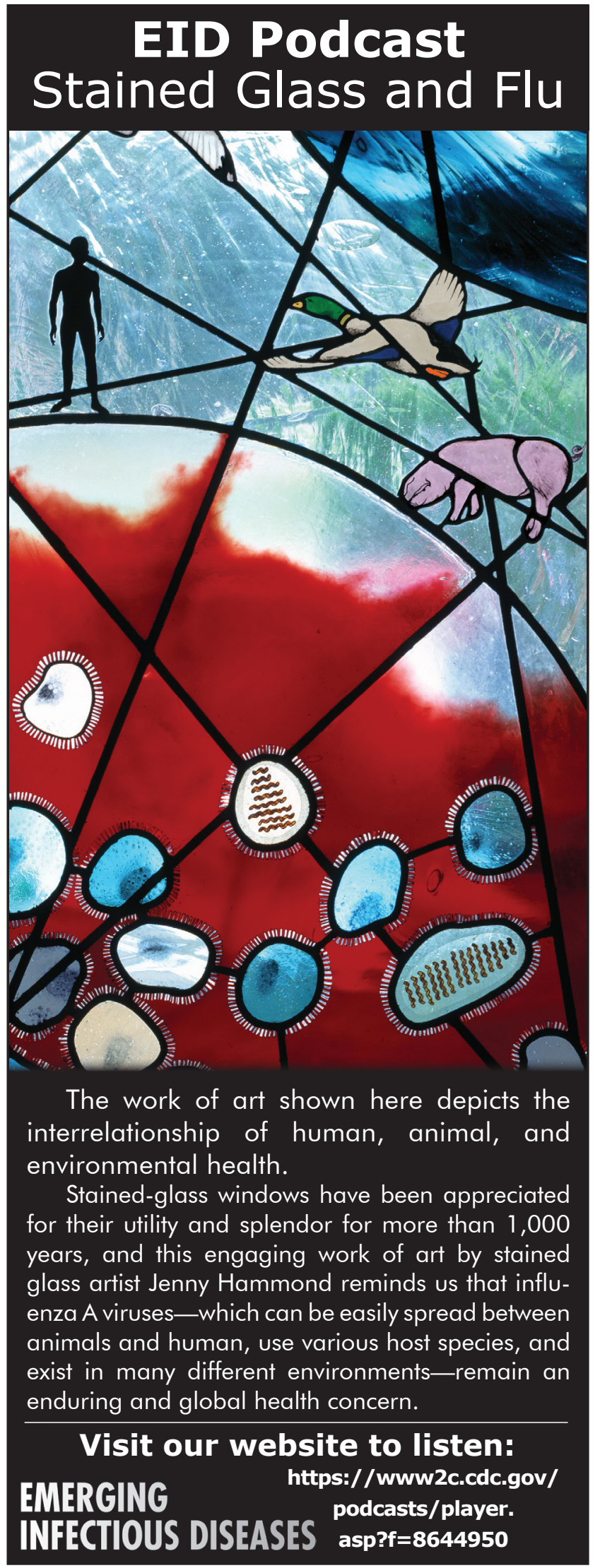

